\title{
AN INTRINSIC CHARACTERIZATION FOR ZERO-DIAGONAL OPERATORS
}

\author{
PENG FAN AND CHE-KAO FONG
}

(Communicated by Palle E. T. Jorgensen)

\begin{abstract}
The purpose of this paper is to present the following intrinsic characterization for zero-diagonal operators.

Theorem. An operator $T$ has a zero diagonal if and only if $\operatorname{tr} \operatorname{Re}\left(e^{i \theta}\right)_{+}=$ $\operatorname{tr} \operatorname{Re}\left(e^{i \theta} T\right)$ - for all $\theta, 0 \leq \theta<2 \pi$.
\end{abstract}

A bounded linear operator $T$ on a complex separable Hilbert space $H$ is said to have a zero diagonal if there is an orthonormal basis $\left\{b_{n}\right\}$ such that $\left(T b_{n}, b_{n}\right)=0$ for all $n$. It is well known that if the dimension of $H$ is finite, then an operator on $H$ has a zero diagonal if and only if its trace is zero; see, e.g., [3, p. 109]. This was generalized to operators on Hilbert spaces in the following form.

Theorem A [1, Theorem 1]. An operator $T$ has a zero diagonal if and only if there exists an orthonormal basis $\left\{b_{n}\right\}$ such that the sequence $\left\{s_{n}\right\}$ of partial sums of the diagonal entries

$$
s_{n}=\sum_{k=1}^{n}\left(T b_{k}, b_{k}\right)
$$

has a subsequence converging to zero.

The above characterization is very sensitive to a base change. However, it can be reformulated into base free descriptions in special cases. For instance, it reduces to $\operatorname{tr} T=0$ if $T$ is a trace class operator. When $T$ is hermitian, it can be converted into the following.

Theorem B [2, Theorem 2]. A hermitian operator $H$ has a zero diagonal if and only if $\operatorname{tr} H_{+}=\operatorname{tr} H_{-}$. tion.

The purpose of this paper is to present the following intrinsic characteriza-

Received by the editors October 12, 1992.

1991 Mathematics Subject Classification. Primary 47A99; Secondary 47A12.

Key words and phrases. Zero diagonal operators, trace, trace-class operators. 
Theorem. An operator $T$ has a zero diagonal if and only if

$$
\operatorname{tr} \operatorname{Re}\left(e^{i \theta} T\right)_{+}=\operatorname{tr} \operatorname{Re}\left(e^{i \theta} T\right)_{-}
$$

for all $\theta, 0 \leq \theta<2 \pi$.

Throughout this paper, $\operatorname{Re} T$ denotes the real part of $T ; H_{+}$and $H_{-}$stand for $(|H|+H) / 2$ and $|H|-H_{+}$respectively where $H$ is hermitian; and $\operatorname{tr} P$ means the trace of a positive operator $P$. In addition, we denote by $R\{\operatorname{tr} T\}$ the set of all sums $\sum\left(T b_{n}, b_{n}\right)$ whenever the series converges with respect to some orthonormal basis $\left\{b_{n}\right\}$.

The proof of our Theorem relies primarily on the following result about the "shape" of $R\{\operatorname{tr} T\}$.

Theorem C [2, Theorem 4]. For an operator $T, R\{\operatorname{tr} T\}$ is either empty, or a point, or a line, or the complex plane. Precisely:

(i) $R\{\operatorname{tr} T\}=\varnothing$ iff $\operatorname{tr} \operatorname{Re}\left(e^{i \theta} T\right)_{-}<+\infty$ but $\operatorname{tr} \operatorname{Re}\left(e^{i \theta} T\right)_{+}=+\infty$ for some $\theta$.

(ii) $R\{\operatorname{tr} T\}$ is a point iff $\operatorname{tr} \operatorname{Re}\left(e^{i \theta} T\right)_{+}<+\infty$ for all $\theta$.

(iii) $R\{\operatorname{tr} T\}$ is a line iff $\operatorname{tr} \operatorname{Re}\left(e^{i \theta} T\right)_{ \pm}<+\infty$ and $\operatorname{tr} \operatorname{Im}\left(e^{i \theta} T\right)_{ \pm}=+\infty$ for some $\theta$.

(iv) $R\{\operatorname{tr} T\}$ is the complex plane iff $\operatorname{tr} \operatorname{Re}\left(e^{i \theta} T\right)_{+}=+\infty$ for all $\theta$.

Proof of Theorem. Necessity. Suppose $T$ has a zero diagonal. Then obviously $0 \in R\{\operatorname{tr} T\}$ and thus $R\{\operatorname{tr} T\}$ takes only three shapes by Theorem C. When $R\{\operatorname{tr} T\}=\{0\}, T \in C_{1}$, the trace class; hence $\operatorname{tr} \operatorname{Re}\left(e^{i \theta} T\right)_{ \pm}=0$. When $R\{\operatorname{tr} T\}$ is a line containing 0 , by (iii) of Theorem $C$, there is a $\theta$ such that $\operatorname{Re}\left(e^{i \theta} T\right) \in C_{1}$ and $\operatorname{tr} \operatorname{Im}\left(e^{i \theta} T\right)_{ \pm}=+\infty$. This implies $\operatorname{tr} \operatorname{Re}\left(e^{i \phi} T\right)_{ \pm}=+\infty$ for $e^{i \phi} \neq e^{i \theta}$ and $e^{i(\theta+\pi)}$. Lastly when $R\{\operatorname{tr} T\}$ is the plane, $\operatorname{tr} \operatorname{Re}\left(e^{i \theta} T\right)_{+}=+\infty$ for all $\theta$.

Sufficiency. If $\operatorname{tr} \operatorname{Re}\left(e^{i \theta} T\right)_{+}=\operatorname{tr} \operatorname{Re}\left(e^{i \theta} T\right)_{-}$for all $\theta, R\{\operatorname{tr} T\}$ acquires the same three shapes. It is enough to show $0 \in R\{\operatorname{tr} T\}$ in each case because this proves that $T$ has a zero diagonal, by Theorem $\mathrm{A}$. When $R\{\operatorname{tr} T\}$ is the complex plane, obviously it contains 0 . When $R\{\operatorname{tr} T\}$ is a point, the hypothesis implies that $\operatorname{tr} T=0$. Finally when $R\{\operatorname{tr} T\}$ is a line, by (iii) we can write $e^{i \theta} T=$ $H+i K$ such that $\operatorname{tr} H_{ \pm}=+\infty$ and $\operatorname{tr} K_{+}=\operatorname{tr} K_{-}<+\infty$. Now according to Theorem B, $H$ has a zero diagonal with respect to some orthonormal basis $\left\{b_{n}\right\}$. Thus

$$
\sum\left(T b_{n}, b_{n}\right)=e^{-i \theta} \sum\left(i K b_{n}, b_{n}\right)=0 .
$$

This shows $0 \in R\{\operatorname{tr} T\}$ and completes the proof.

Remarks. (i) We point out here that not a single $\theta$ can be omitted from the hypothesis in the proof of the sufficiency part above. Indeed, define $T=$ $\operatorname{diag}\left\{1,-1, i, \frac{1}{2},-\frac{1}{2}, \frac{i}{2}, \ldots, \frac{1}{n},-\frac{1}{n}, \frac{i}{n}, \ldots\right\}$. Observe that $\operatorname{tr} \operatorname{Re}\left(e^{i \theta} T\right)_{+}=$ $+\infty$ for all $\theta$ except for $\theta=\frac{\pi}{2}$ (in fact, $\operatorname{tr} \operatorname{Re}(i T)_{+}=0$ ). But this operator does not have a zero diagonal because the imaginary part is positive.

(ii) We provide here an alternative proof for the necessity part. Suppose $\left(T b_{n}, b_{n}\right)=0$, for all $n$, with respect to an orthonormal basis $\left\{b_{n}\right\}$. Write $H$ for $\operatorname{Re}\left(e^{i \theta} T\right)$. Obviously $\left(H b_{n}, b_{n}\right)=0$ for all $n$. Hence

$$
\operatorname{tr} H_{+}=\sum\left(H_{+} b_{n}, b_{n}\right)=\sum\left(\left(H+H_{-}\right) b_{n}, b_{n}\right)=\sum\left(H_{-} b_{n}, b_{n}\right)=\operatorname{tr} H_{-} .
$$




\section{REFERENCES}

1. P. Fan, On the diagonal of an operator, Trans. Amer. Math. Soc. 283 (1984), 239-251.

2. P. Fan, C.-K. Fong, and D. A. Herrero, On zero-diagonal operators and traces, Proc. Amer. Math. Soc. 99 (1987), 445-451.

3. P. R. Halmos, Finite-dimensional vector spaces, Van Nostrand, Princeton, NJ, 1958.

Department of Mathematics, Texas Christian University, Ft. Worth, Texas 761290001

Department of Mathematics, Carleton University, Ottawa, Ontario, Canada K1S 5B6 